\title{
Measurements of Mechanical $Q$ in Levitated Paramagnetic Crystals
}

\author{
S. J. Augst and R. W. P. Drever \\ Department of Physics, Mathematics, and Astronomy \\ California Institute of Technology, Pasadena, CA 91125
}

\begin{abstract}
Thermal noise from test masses, arising both from internal noise in the test mass material and from losses in the suspension wires and their attachments, is a significant factor limiting sensitivity of interferometric gravity-wave detectors. To investigate ways of reducing these noise sources we are using magnetic levitation in place of suspension wires. A search for high-Q crystals with magnetic properties allowing tests in moderate field strengths has led us to paramagnetic crystals, and we report preliminary results with small levitated samples of Gadolinium Gallium Garnet (GGG) and Terbium Gallium Garnet (TGG). The technique seems the first to allow $Q$ measurements with no mechanical contact, and may facilitate work aimed at reducing thermal noise.
\end{abstract}

\section{INTRODUCTION}

Interferometric gravitational-wave detectors may experience noise from a number of sources, several of which are due to noise from wires or fibers used to suspend and isolate the test masses, or are transmitted by these from the surroundings to the test masses. In the program of which the present work is a part, magnetic levitation of test masses is being investigated as a possible alternative to wire or fiber suspensions. Potential benefits that might be obtained include: 1) the possibility of achieving lower suspension resonance frequencies, 2) elimination of "violin mode" resonances of wires or fibers, 3) improved seismic isolation, 4) possible reduction of suspension thermal noise, and 5) avoidance of damping of internal modes of the test mass by the wires or fibers.

There are some potential disadvantages also, including possibilities of other noise sources, and these are being investigated. In earlier experiments ${ }^{1}$ we have levitated test masses by attaching to them non-conducting ceramic permanent magnets, and applying lifting forces by interaction with fixed ceramic permanent magnets located above them. Relatively long pendulum-mode relaxation times ( $\sim 15$ hours) were achieved, and long suspension periods ( $\sim 8$ seconds or more). However the ceramic magnets on the test masses have higher mechanical damping than typical test mass materials, and this could lead to significant internal thermal noise unless there is effective isolation between the magnets and the test masses themselves. It would be preferable to find materials which themselves combine high mechanical quality factors, $Q$, with sufficiently strong magnetic properties to make levitation practicable without extreme magnetic fields, and

CP523, Gravitational Waves: Third Edoardo Amaldi Conference, edited by S. Meshkov (C) 2000 American Institute of Physics 1-56396-944-0/00/\$17.00 
use these to obtain low thermal noise for both pendulum and internal modes. We have been looking for suitable materials for several years, and recently have been considering paramagnetic crystals, which might also possess good optical qualities. Two materials which we found to give encouraging results in preliminary $Q$ tests using piezoelectric transducers were Terbium Gallium Garnet (TGG) and Gadolinium Gallium Garnet (GGG). ${ }^{2}$ These materials are used commonly in optical Faraday rotators.

\section{EXPERIMENTAL PROCEDURE AND RESULTS}

Here we report on mechanical Q measurements made with cylindrical samples of TGG and GGG, $15 \mathrm{~mm}$ in diameter and $8 \mathrm{~mm}$ thick. It was found that with these small samples there could be serious damping from suspension wires and piezoelectric drivers, so we have developed and used a levitation technique with non-contacting excitation and sensing of internal resonances. A simplified diagram of the arrangement is shown in Figure 1.

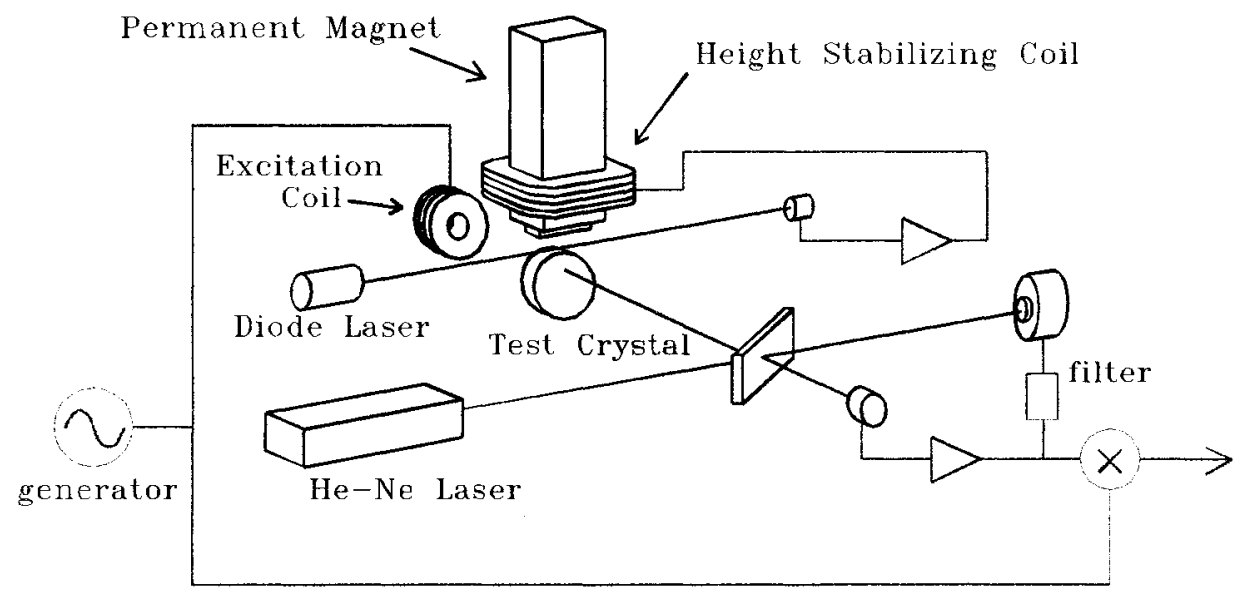

FIGURE 1. Experimental setup for $Q$ measurement. The cylindrical paramagnetic sample is suspended in the field of a permanent magnet, with its height stabilized using a shadow sensor whose output is fed back to a coil which adjusts the field. A mechanical resonance in the sample is excited by another coil and the resulting crystal vibration is sensed with the coherently demodulated signal from a Michelson interferometer. Light in one arm of the interferometer is reflected by an uncoated surface of the sample, while a mirror in the other arm is servo-controlled to lock the interferometer to a single fringe. Most of the system is in vacuum.

The paramagnetic sample is placed in the field of a permanent magnet which creates an induced magnetism in the crystal which interacts with the field gradient to provide lift. The induced magnetism makes it convenient to excite resonances in the sample by a high-frequency current in an adjacent coil, whose frequency is scanned through the resonances, while the vibrations are sensed with a Michelson interferometer. A short focus "cats-eye" lens (not shown) and a slow servo-controlled mirror makes the signal insensitive to misalignment and slow motions of the levitated crystal. For precise $Q$ 
determination the excitation coil can be suddenly disconnected and the decay of the undriven signal measured. This technique provides a means to measure the $\mathrm{Q}$ of a sample in a totally non-contacting way, with minimal external damping.

To maintain stable levitation the output of a shadow sensor is fed back to a height stabilizing coil which adjusts the field of the permanent magnet. The upward force on a paramagnetic sample is proportional to $B_{z} \times \chi_{m} \times d B_{z} / d z$ where $B_{z}$ is the vertical component of the magnetic field, and $\chi_{m}$ is the paramagnetic susceptibility. Typical products of field times field gradient in these samples were approximately $7 \mathrm{kGauss}^{2} / \mathrm{cm}$ for TGG and $10 \mathrm{kGauss}^{2} / \mathrm{cm}$ for GGG. Rare-earth magnets were used to obtain the fields in these measurements. These magnets are electrically conductive, and thus could be a source of damping from eddy currents. Checks of the pendulum mode relaxation time with these samples indicate, however, that in our present situation and for the levels of $Q$ found here eddy current damping is not a significant factor.

The crystals used in the present experiment are cylindrical in shape and initially had an inspection polish on the two flat surfaces with a fine grind finish on the circumference. Under these conditions the value for $Q$ found for the GGG sample was $6.7 \times 10^{5}$ at a resonance frequency of $221.5 \mathrm{kHz}$. It was expected that the ground finish was limiting $\mathrm{Q}$, and the same sample was subsequently repolished after the Meeting, and was then found to give significantly higher $Q .{ }^{3}$

The TGG sample available initially had a small visible crack in its interior and a chip on the exterior. With this sample we obtained a $Q$ value of $3.7 \times 10^{4}$ at $219.6 \mathrm{kHz}$. After the Meeting, we obtained an undamaged and finer-polished sample of TGG with the same dimensions, and this also gave a significantly higher value for $Q{ }^{3}{ }^{3}$ It is clear that further work is required, and these preliminary results should only be regarded as encouraging lower limits to the material $Q$ values.

\section{POTENTIAL APPLICATIONS AND FURTHER WORK}

The long term aim in this research has been to find ways of suspending test masses with reduced thermal noise. Present results are encouraging, at least for small test masses, but further work is needed for larger masses since high fields and gradients are required. In the long term this technique may prove to be particularly convenient at cryogenic temperatures where paramagnetic susceptibilities are significantly higher (e.g. $\chi_{m}$ is about 30 times larger at $10 \mathrm{~K}$ than at room temperature).

A potentially more immediate possibility is the use of paramagnetic crystals as replacements for the orientation and length control magnets that are currently attached to test masses in interferometers. The initial LIGO test mass Q's are damped by the low-Q nature of these magnets and they must not be present in the LIGO II upgrade if it is to achieve its projected noise levels. Potential control technologies that have been suggested are electrostatic transducers or photon pressure from a laser. A possible alternative solution is to bond high-Q paramagnetic crystals to the test masses and use conventional coil drivers to provide driving forces. It is unlikely that this arrangement would damp the internal test mass $\mathrm{Q}$. Additionally the control coils could be operated with a high-frequency driving field (rather than a DC field as is required with 
permanent magnets) thus making the test masses less prone to noise from external magnetic field fluctuations (e.g. from the earth's field or from electrical equipment).

As far as we know these experiments are the first measurements of high $Q$ single crystals made without any mechanical contact. This may provide a means of achieving higher values of $Q$ than possible with any other arrangement. Comparison of results using this technique with results from conventional wire suspensions may be useful in studies to identify practical sources of loss.

The application of this measurement technique to larger samples will be used to determine values of $Q$ for lower frequencies. In addition samples that are superpolished on all surfaces will provide further insight into the importance of surface losses versus bulk material losses. Larger crystals require a larger volume of high magnetic field so an electromagnet is now being used to levitate these. Further tests should show whether TGG or GGG crystals could be competitive alternative test mass materials to fused silica or perhaps even to sapphire. Unfortunately, levitation of these crystals requires large magnetic fields and scaling the crystal samples up to LIGO-sized test masses might require superconducting magnets for levitation, and this is an area that requires further study.

The techniques of magnetic levitation for these $Q$ measurements is important in itself since it provides the possibility of studying suspension losses. A comparison of $Q$ measurements between a fiber suspended mass and the same mass suspended magnetically might provide important insights into the loss mechanisms present in conventional LIGO suspension designs. Additionally, this technique could prove to be useful for small, very-low noise systems such as for QND experiments, and these ideas are being considered further.

\section{ACKNOWLEDGMENTS}

We would like to acknowledge valuable discussions with J. L. Hall and R.E. Cowen, and H. J. Kimble for some early material samples. This research is based upon work supported by the National Science Foundation under Grant number PHY-9722112, and the California Institute of Technology.

\section{REFERENCES}

1. Drever, R. W. P. "Some New Concepts for Laser Interferometer Gravitational Wave Detectors," Proceedings of the Moriond Workshop on Dark Matter \& Cosmology, Quantum Measurements and Experimental Gravitation, Les Arcs, January 1996, ed. J. Tran Thanh Van (Editions Frontieres, 1996).

2. These particular materials were suggested to us by J. L. Hall (JILA).

3. New results have been obtained since the conference took place. A new polished TGG crystal was obtained and the existing GGG crystal was polished. Both faces, as well as the circumference of each crystal were polished to $60 / 40 \mathrm{scratch} / \mathrm{dig}$ and $\lambda / 1$. Measured values of $Q$ for the polished crystals were $6.0 \times 10^{6}$ and $9.7 \times 10^{6}$ respectively. It is anticipated that a "super polish" would raise the values of $Q$ even further. 